\title{
The Influence of an Applied Heat Flux on the Violence of Reaction of an Explosive Device
}

\author{
Charles A. Wight \\ Department of Chemistry \\ University of Utah \\ Salt Lake City, Utah \\ chuck.wight@utah.edu
}

Monica Hall

Department of Chemistry

University of Utah

Salt Lake City, Utah

hall@chem.utah.edu

\author{
Jacqueline C. Beckvermit \\ Department of Chemistry \\ University of Utah \\ Salt Lake City, Utah \\ j.beckve@chem.utah.edu
}

\author{
Todd Harman \\ Department of Mechanical \\ Engineering \\ University of Utah \\ Salt Lake City, Utah \\ t.harman@utah.edu
}

\author{
Martin Berzins \\ Scientific Computing and \\ Imaging Institute \\ University of Utah \\ Salt Lake City, Utah \\ mb@sci.utah.edu
}

\begin{abstract}
It is well known that the violence of slow cook-off explosions can greatly exceed the comparatively mild case burst events typically observed for rapid heating. However, there have been few studies that examine the reaction violence as a function of applied heat flux that explore the dependence on heating geometry and device size. Here we report progress on a study using the Uintah Computation Framework, a high-performance computer model capable of modeling deflagration, material damage, deflagration to detonation transition and detonation for PBX9501 and similar explosives. Our results suggests the existence of a sharp threshold for increased reaction violence with decreasing heat flux. The critical heat flux was seen to increase with increasing device size and decrease with the heating of multiple surfaces, suggesting that the temperature gradient in the heated energetic material plays an important role the violence of reactions.
\end{abstract}

\section{Categories and Subject Descriptors}

D.4 [Mathematics of Computing]: Mathematical Software; J.2 [Computer Applications]: Physical Sciences and Engineering; D.1.3 [Software]: Concurrent Programming

\section{General Terms}

Experimentation, Theory

\section{Keywords}

Deflagration, Detonation, DDT, Cook-off, Violence of Reaction

Permission to make digital or hard copies of all or part of this work for personal or classroom use is granted without fee provided that copies are not made or distributed for profit or commercial advantage and that copies bear this notice and the full citation on the first page. Copyrights for components of this work owned by others than ACM must be honored. Abstracting with credit is permitted. To copy otherwise, or republish, to post on servers or to redistribute to lists, requires prior specific permission and/or a fee. Request permissions from Permissions@acm.org.

XSEDE'13 July 22 - 25 2013, San Diego, CA, USA.

Copyright 2013 ACM 978-1-4503-2170-9/13/07...\$15.00.

\section{INTRODUCTION}

Cook-off is a non-shock event that involves bulk heating of a confined or unconfined explosive device to the point of ignition [9]. The rate of heating determines if it is a fast or slow cook-off. The cook-off response of an energetic material is a vital topic in munitions design, and in the transportation and storage of energetic devices[3]. As experiments to study these hazards are very costly and can be inconclusive, computer modeling can help predict behaviors under certain conditions, especially cook-off scenarios. Location of the point of ignition has been identified as a dominate factor in the violence of the reaction and it will occur where the heat source from the exothermic reaction exceeds the thermal diffusion rate [18]. When the energetic material is externally heated quickly (fast cook-off), the heat is slow dissipating through the solid causing a large temperature gradient. The relatively low thermal conductivity means that it behaves as an insulator, so the points of ignition originate on the surface. Fast cook-off experiments have been found to cause mild explosions due to a rapid pressure increase at the surface, bursting the confinement structure without consuming all of the explosive. During a slow heating process (slow cook-off), heat is conducted further into the explosive forming a uniform temperature distribution in the energetic material. In a slow cook-off, the point of ignition usually occurs near the center of the device and the explosion is observed to be very violent and may lead to detonation $[29,2$, 32 . It is generally accepted that a slow cook-off leads to a more violent explosion than fast cook-off.

The violence of a thermal explosion is dependent upon numerous variables and conditions including the mechanical and thermal properties of the energetic material, the deflagration behavior, heating rate, and the degree of confinement $[29,18]$. These variables factor into the reaction rate which occurs after ignition of the energetic material. The violence of reaction can be measured by the number of fragments (size and velocity) produced, amount of explosive material consumed, overpressure at some distance from the center of the device, the confinement strain, kinetic energy, or the amount and rate of energy being released [26, 24]. For 
an event to be "violent", the pressure generated from the solid $\rightarrow$ gas reaction must exceed the yield strength of the confinement structure. The pressure must then continue to rise at a rate fast enough that the confinement and gas cannot move appreciably [24]. The violence of reaction increases until a) the confinement is breached, b) energetic material is consumed, or c) a transition to a different reaction regime such as detonation occurs [18].

Many cook-off studies have been performed to quantify the violence of reaction under various conditions. The One Dimensional Time to Explosion test (ODTX) [6] was performed on energetic spheres encased in preheated aluminum to develop the kinetics of thermal ignition and predict the time to explosion for a given temperature history. The Scaled Thermal Explosion Experiment (STEX) [31] and Variable Confinement Cook-Off Tests (VCCT) [1] were very similar. They subjected a charge to a heating rate and measure the velocity of the steel enclosure along with the temperature profile in side of the energetic material. These tests varied the thickness of the enclosure and the heating rates and compared the violence of reaction under controlled conditions $[18,1]$. Several proposed deflagration to detonation mechanisms have been suggested including the coalescing of pressure waves to form a shock front that exceeds the pressure threshold for detonation [4], detonation ignition produced by a gradient of reactivity [15], and the formation of a temperature gradient providing stimuli for the bulk material [7].

While experiments have focused on the effects of the confinement of the reactant and heating rates little is known about the effect of device size and heating geometry on the violence of reaction. The purpose of this study is to computationally measure the violence of reaction of several PBX9501 (95\% HMX, 2.5\% Estane, and 2.5\% BDNPA-F) filled devices encased in steel, subjected to a range of heat fluxes using Uintah [10]. The focus is determining the threshold heat flux for a mild violence of reaction (such as a case burst during a fast cook-off) or an extremely violent reaction (such as a detonation during a slow cook-off) and proposing mechanisms for a deflagration to detonation transition. The mechanism of deflagration to detonation transition has yet to be fully understood. Under adiabatic conditions the deflagration of PBX9501 will reach pressures around $2 \mathrm{GPa}$ while pressures over 5 GPa are required for detonation [25]. This leads to the interest of understanding how a fundamentally metastable process can create pressures exceeding detonation thresholds and sustain steady state detonation around $35 \mathrm{GPa}$.

\section{SIMULATION METHODOLOGY 2.1 Uintah Computation Framework}

The MPM-ICE algorithm [11] is used to solve the governing equations for our reacting fluid-structure-interaction (FSI) problem and is implemented in Uintah as a "component." In addition to the MPM-ICE algorithm, Uintah integrates numerous sub-components including equations of state, constitutive models and solid $\rightarrow$ gas reaction models. This FSI technique is unique in that a Lagrangian particle method for doing solid mechanics computations is integrated with a general multi-material computational fluid dynamics (CFD) formulation. In the "multi-material" CFD approach, each material (either fluid or solid) is defined at the continuum level over the entire computational domain, including regions where a material does not exist. In addition to the physical state, (i.e., mass, momentum, energy) at each discrete point, the volume fraction of each material is tracked with the constraint that the volume fractions of all materials must sum to unity in any grid cell $[14,12,13]$.

To solve the discretized multi-material equations we use a cell-centered formulation of the ICE (for Implicit, Continuousfluid, Eulerian) algorithm [14]. The use of a cell-centered, finite volume solution technique is convenient in that a single control volume is used for all materials, simplifying the solution of the integral conservation equations of mass, momentum and energy and the exchange of these quantities between the materials. The ICE method is fully compressible and has also recently been extended to high speed flows [30], an important consideration in simulations involving explosions of any type, particularly detonations. In addition to the source terms present in any CFD formulation, the multi-material equations include exchange terms for mass, momentum and heat. Inter-material mass exchange is based on solid $\rightarrow$ gas reaction models. Momentum and heat exchange is typically modeled as a drag law based on relative material velocities or temperatures, respectively. These velocities and temperatures are computed in a point-wise implicit manner to avoid stability considerations and ensure conservation.

The multi-material ICE formulation was initially developed for the solution of multi-phase flow phenomena, and as such, no explicit distinction is made between fluid and solid materials in the model equations. Fluid-solid interfaces are not tracked, nor are boundary conditions passed through them $[14,12]$. To maintain the integrity of the fluid-solid interface and provide a mechanism to track the deformation history of the solid(s) we employ the Material Point Method (MPM). This method is used to evolve the equations of motion for the solid materials and is a powerful technique for doing computational solid mechanics [5, 28]. Lagrangian particles or material points are used to discretize the volume of a material and each particle carries state information (e.g. mass, volume, velocity, and stress) about the portion of the volume that it represents. Our implementation uses a Cartesian grid as a computational scratchpad for computing spatial gradients, the same grid used by the multi-material CFD component. During each timestep, the state of the particles is projected to the computational grid. The states of all materials, fluid and solid, are computed, and in the case of the solid materials, changes in state are interpolated back to the particles. This change in the particle state includes the position, velocity, temperature, and mass in regions where there are solid to gas reactions.

The methods described above are implemented as components inside of Uintah. The core of Uintah is a sophisticated computational framework that can integrate multiple simulation components, analyze the dependencies and communication patterns between them, and execute the resulting multi-physics simulation [23]. Uintah utilizes an abstract representation (called a task-graph) of parallel computation and communication to express data dependencies between multiple physics components. The task-graph is a directed 

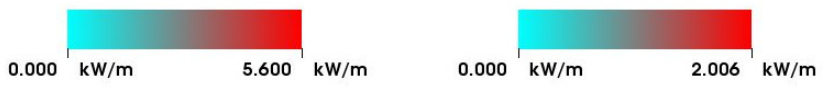

(a)

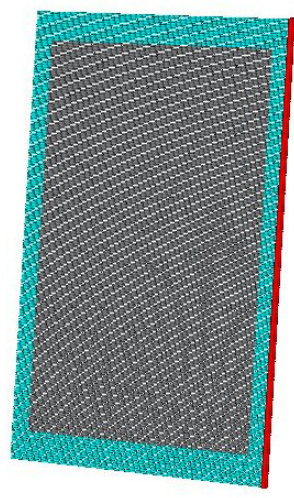

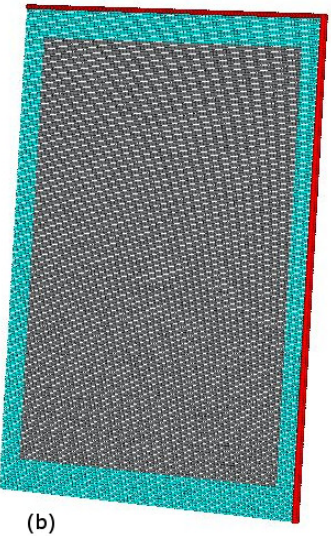

(b)
Figure 1: A 2D particle visual of an $80 \times 80 \times 1 \mathrm{~mm}$ PBX9501 (grey material) device with $6 \mathrm{~mm}$ steel casing subject to a heat flux on (a) 1 side and (b) 2 sides. Note only the first layer of steel particles are experiencing any heat flux.

acyclic graph of tasks. Each task consumes some input and produces some output (which is in turn the input of some future task). Using this task-graph and domain decomposition, the scheduler component creates an execution schedule with an execution order and the required MPI communication. For each timestep, this schedule is then executed repeatedly. Within a timestep, the scheduler component is able to dynamically assign tasks to available computational resources on-node, including CPU cores and GPUs. The task-graph allows Uintah to analyze the structure of the computation, to automatically enable load-balancing, data communication, parallel I/O, and checkpointing. Improvements to this task-graph have focused on implementing out of order execution to improve machine utilization [22].

A load balancer component is responsible for assigning each hexahedral mesh patch to a processor. Uintah's load balancer utilizes space-filling curves in order to cluster patches together [17]. Accurate workload prediction is problematic with adaptive meshes. The complexity of the underlying physics complicates the process of deriving an accurate cost model. For example, MPM simulations have large numbers of computationally expensive particles that move throughout the domain which may cause the workload to change at every timestep. To address this imbalance a technique has been developed which uses forecasting methods to predict the cost of each patch based on observations made at runtime. During task execution, the time to complete each task is recorded and used to update a simple forecasting model which is then used to predict the time to execute on each patch in the future. This provides a mechanism to accurately predict the cost of each patch while requiring little information from the user or component developer [16].

Uintah demonstrates good strong and weak scaling using the core algorithm described above. More information on the performance and scalability of Uintah are described in $[19,20,27,21]$.
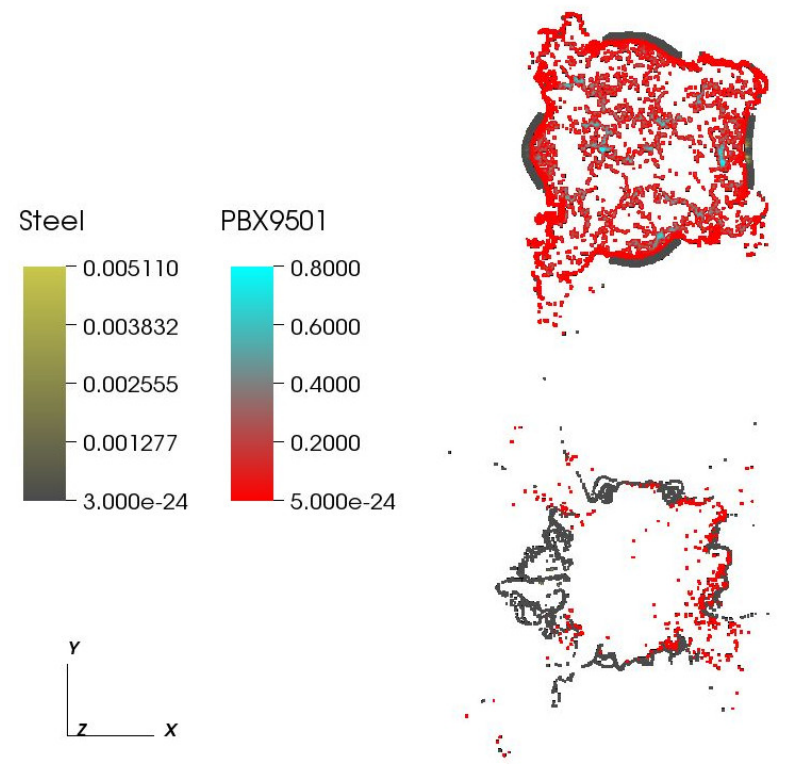

Figure 2: Volume fraction of each material per cell for the different cook-off responses. The top image shows a pressure burst and the bottom a detonation.

\subsection{Simulation Setup}

The explosive devices studied are 2-dimensional boxes using $1 \mathrm{~mm}$ grid cell size resolution with symmetric boundaries in the $z$ directions. Excess space was added to the computational domain in the $x$ and $y$ direction to allow the device to expand and the maximum kinetic energy to be calculated without a loss of mass. The energetic material (PBX9501) was modeled using an experimentally validated JWL equation of state, a ViscoSCRAM damage model, Ward, Son and Brewster burn rate model and JWL ++ detonation model [25]. The square energetic devices were confined with $6 \mathrm{~mm}$ of steel represented by an elastic-plastic constitutive model [8]. The device sizes ranging from $80^{2} \mathrm{~mm}^{2}$ to $100^{2} \mathrm{~mm}^{2}$. Simulations were run on NICS's Kraken machine.

Each simulation consisted of three phases: a heat up phase, ignition/reaction phase, and the event/cook-off response. The heat up phase consisted of a heating flux applied to the outside of the steel container on one or two sides seen in Figure 1. The steel and PBX9501 particle resolution is 8 particles $/ 1 \mathrm{~mm}^{3}$ and the flux was calculated in units of watts/particle or $k W / m^{2}$. The device was heated to an ignition temperature of $550 \mathrm{~K}$ from an initial temperature of $300 K$. After ignition, pressure rapidly builds in the device due to product gas formation. The cook-off response ranged from a pressure burst (confinement was breached with low damage), a mild explosion due to excess deflagration (confinement and energetic material is extensively fragmented), to a violent explosion (typically detonation). Figure 2 shows the responses observed in this study. The top image shows the volume fraction of PBX9501 and the steel container after a pressure burst. This simulation was run on a $80 \mathrm{~mm} \mathrm{x}$ $80 \mathrm{~mm} \times 1 \mathrm{~mm}$ device with a heat flux of $4.8 \mathrm{~kW} / \mathrm{m}^{2}$ on one side. After the case burst there was $81.3 \%$ of the reactant remaining. The bottom image shows a violent reaction where detonation occurred. This simulation was run on a device $85 \mathrm{~mm} \times 85 \mathrm{~mm} \times 1 \mathrm{~mm}$ with one sided heated with a flux 
of $16 \mathrm{~kW} / \mathrm{m}^{2}$. After detonation only $1.4 \%$ of the reactant remained. Violence of reaction is measured by the kinetic energy of the steel.
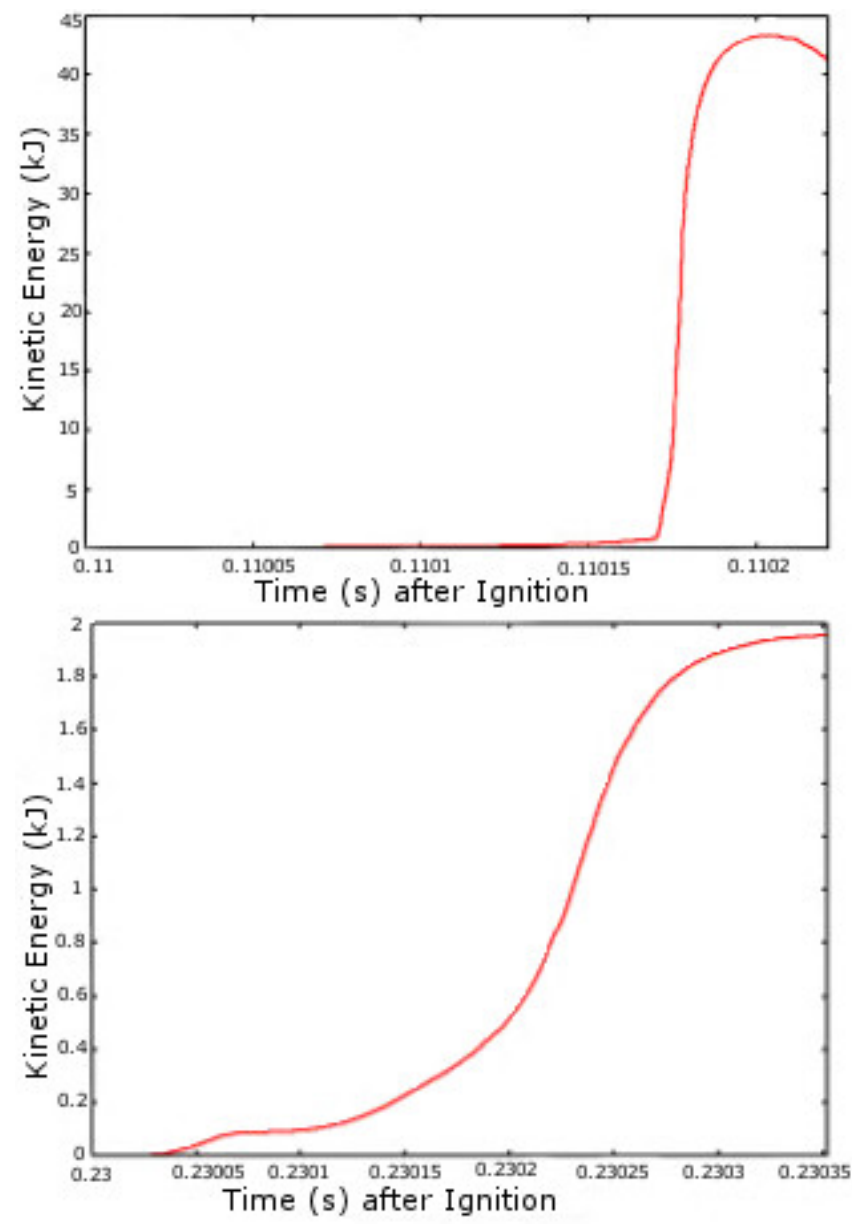

Figure 3: Kinetic energy verses time after ignition of a $100 \times 100 \times 1 \mathrm{~mm}$ (top) charge subject to a $2 \mathrm{~kW} / \mathrm{m}^{2}$ flux on two sides resulting in a detonation. The bottom plot shows the kinetic energy versus time of a $80 \times 80 \times 1 \mathrm{~mm}$ device subject to a $8 \mathrm{~kW} / \mathrm{m}^{2}$ flux on one side resulting in a case burst.

\section{RESULTS AND DISCUSSION}

Each device size was exposed to a wide range of external heat fluxes. With devices smaller than $80^{2} \mathrm{~mm}^{2}$, violent responses were not observed, only mild case bursts. This is due to the structure rupturing and the hot gases escaping before the pressure could build to levels needed for detonation. With these small devices the lack of material being consumed makes the pressure great enough to burst the confinement but not large enough to cause a violent reaction. The kinetic energy of the steel enclosure was monitored over the entire simulation. A sharp increase in the kinetic energy indicates a detonation or a case burst has occurred as seen in Figure 3. At extremely low fluxes, all responses are considered violent, independent of the size and number of sides heated. However as the heating flux increases, there appears to be a distinct difference between event violence at certain heat fluxes varying with device size and number of sides heated.

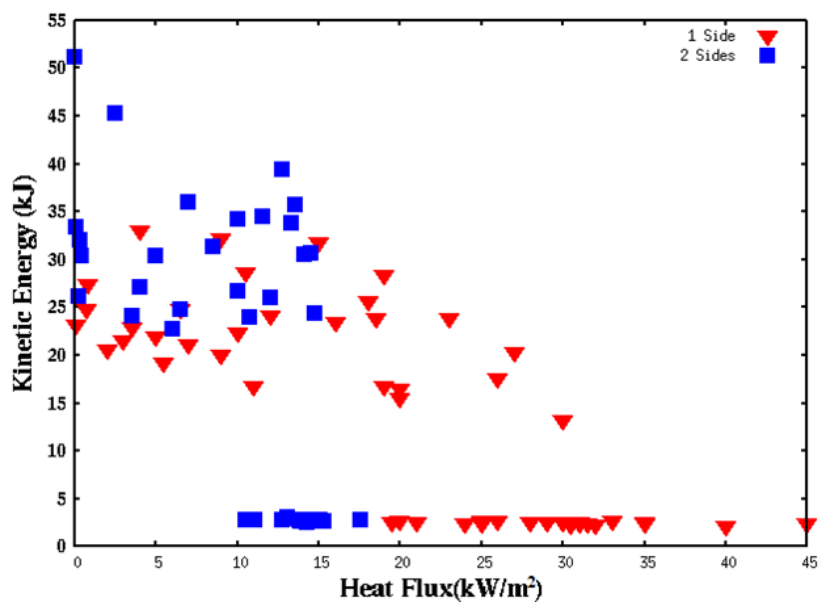

Figure 4: Each point represents the maximum kinetic energy released from a $90 \times 90 \times 1 \mathrm{~mm}$ device subjected to various heat fluxes on one or two sides.

Figure 4 shows the kinetic energy of the steel verses the applied heat flux to a $90 \times 90 \times 1 \mathrm{~mm}$ device. It suggests that as the heat flux increases, there is a sharp change in the kinetic energy between mild explosions and detonation. The higher kinetic energy (10-50 kJ) indicate a (fast) detonation occurring inside of the energetic material. The low kinetic energy $(<5 \mathrm{~kJ})$ is indicative of a (slow) deflagration reaction with case rupture. The critical heat flux is defined as the minimum heat flux in which a case rupture first occurs. The scatter in the data is due to the elastic-plastic material model used for the steel. The material model has a random parameter which causes a variation in the initial ignition site thus changing the strength of the pressure waves. The sensitivity of the pressure waves to coalesce and build pressure within the charge are effected by the initial site of ignition.

There are three proposed mechanisms for a transition from deflagration to detonation: a pressure induced detonation (Figure 5), an ignition site detonation (Figure 6) and a hybrid between the two. The temperature profiles at the time of ignition and during the reaction phase for each mechanism are distinctly different and can provide insight into the different responses. Pressure induced detonation was observed at extremely low heat flux rates and when two sides were heated, while ignition site transition occurs at higher heat fluxes. Figures 5 and 6 show the timelines of these mechanisms leading up to detonation. The upper left colorbar is the temperature of the PBX9501, the lower left is the temperature of the product gas, upper right is the pressure in Pascals with the steel confinement shown in black, and the lower right is the amount of PBX9501 which has reacted during a time-step. The scale is negative because the reactant is being consumed. The pressure and reactant consumption scales change with each frame allowing the pressure waves and variations in reactant consumption to be visualized. The temperature scales for the PBX9501 and product gas remained constant for all images.

The first mechanism presented here, termed pressure induced detonation and seen in Figure 5, occurs at extremely low heat fluxes. During the heat up phase, the device is heated almost uniformly to ignition with a relatively small 

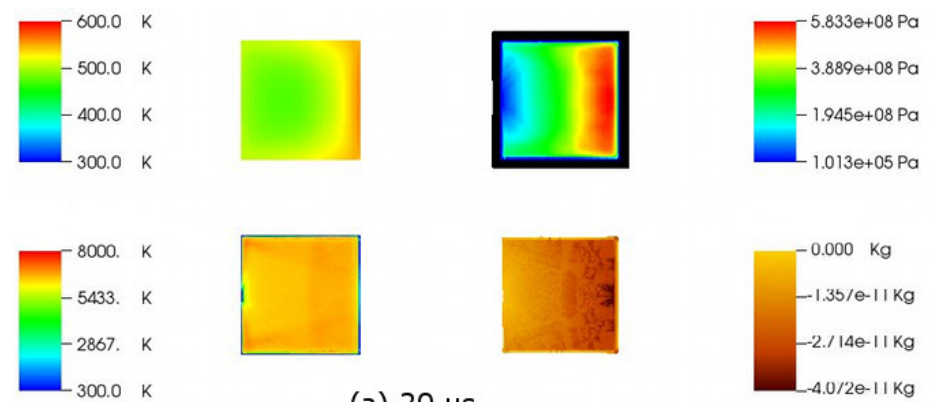

(a) $20 \mu \mathrm{s}$
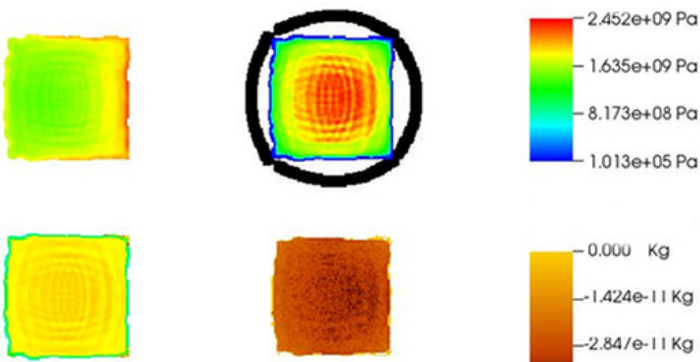

(c) $130 \mu \mathrm{s}$
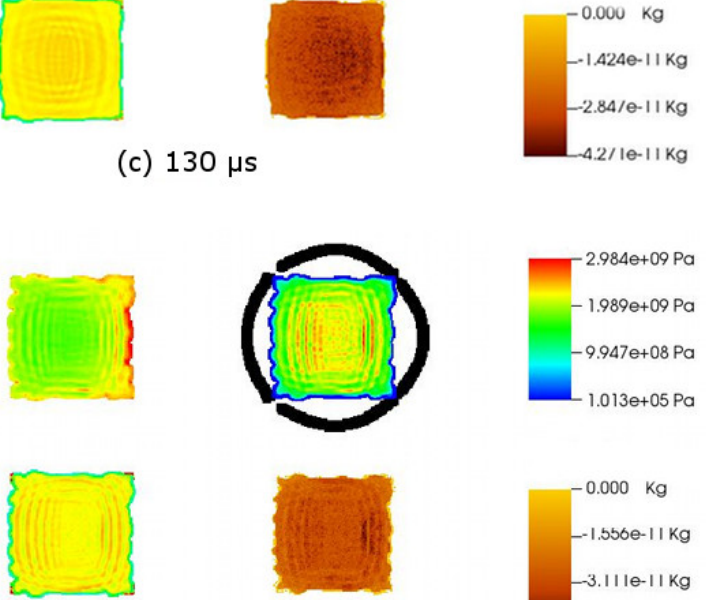

(e) $160 \mu \mathrm{s}$
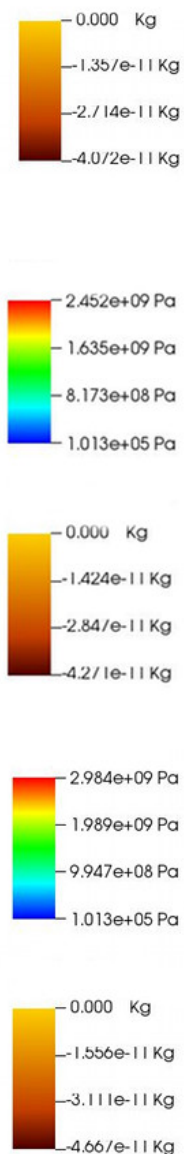

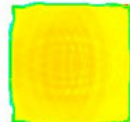

(b) $120 \mu \mathrm{s}$

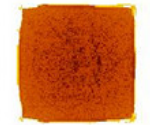

S
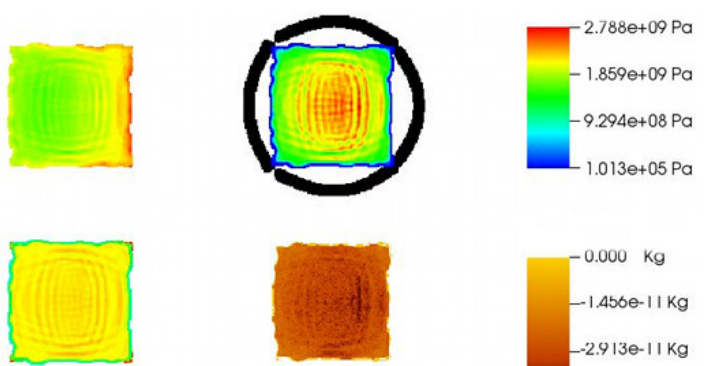

(d) $140 \mu \mathrm{s}$
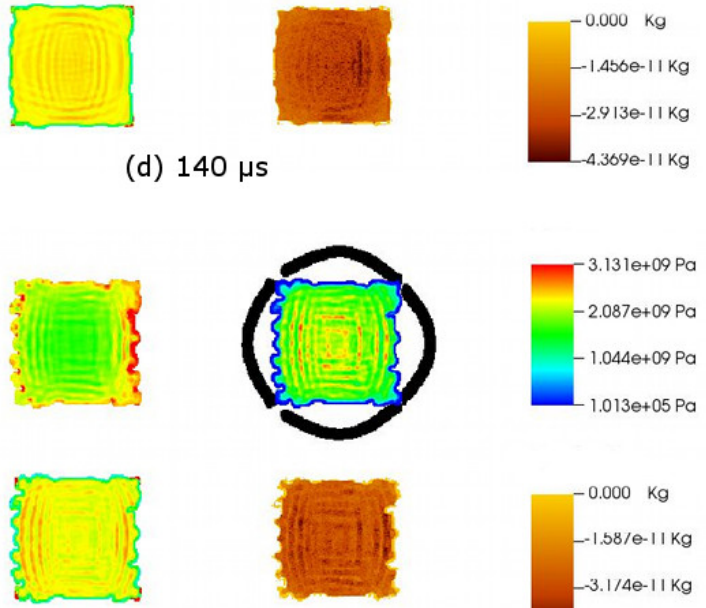

(f) $169 \mu \mathrm{s}$
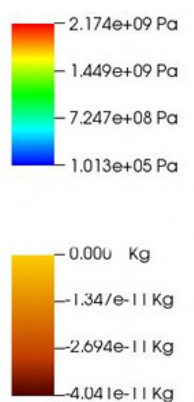

Figure 5: A 90x90x1 $\mathrm{mm}$ device subject to $4 \mathrm{~kW} / \mathrm{m}^{2}$ heat flux on 1 side undergoing a pressure induced detonation. Time reported is the amount of time after ignition.

temperature gradient. It takes about $20 \mu \mathrm{s}$ after ignition for convective deflagration to propagate through the device (Figure 5(a)) and about $70 \mu \mathrm{s}$ for the maximum pressure to be confined to the center of the device. The product gas temperature increases throughout this time but then decreases slightly because the temperature gradient of PBX9501 has not significantly changed, cooling the product gas. This is due to the low thermal conductivity in the condensed phase and the focus is on a short period of time that the material temperature has not been affected significantly. In consequence, the burn rate decreases slightly as shown by the lower right images representing amount of reactant consumed per time-step. However, at $\sim 110 \mu s$ after ignition, there is a significant increase in product gas volume fraction.

The unique characteristic that occurs during this type of detonation is the formation of a grid wave pattern from the product gas within the energetic material having about 4$5 \mathrm{~mm}$ wavelengths (Figure 5(b), $120 \mu \mathrm{s}$ after ignition). This is most noticeable in the pressure profile. About $10 \mu \mathrm{s}$ af- ter the product gas shows this trait, PBX9501 also starts forming the grid pattern (Figure 5(c)). Upon analysis of the velocity vector field of the product gas, it is evident that this grid formation evolves from the gas moving in and out of the condensed phase. Constructive and destructive interference occurs from waves reflecting off the confinement with the waves moving outward producing the grid pattern. The movement of the gas can be due to the higher rate of reaction near the center of the device developing a high product gas concentration. This causes a reaction gradient to form forcing the gas to move outwards. After about $140 \mu \mathrm{s}$ past ignition, the waves become stronger (Figure 5(d-f)) and continue to increase until detonation occurs. This simulation suggests detonation occurs when two waves coalesce and detonation pressures are reached, around $170 \mu \mathrm{s}$ after ignition. The average amount of reactant going to product during a time-step in the reaction phase is $\sim 3 \times 10^{-11} \mathrm{~kg}$ per cell, and in the compaction waves it increases to $\sim 4.6 \times 10^{-11} \mathrm{~kg}$.

The other mechanism observed occurs when two pressure waves from separate ignition sites coalesce and build to reach 

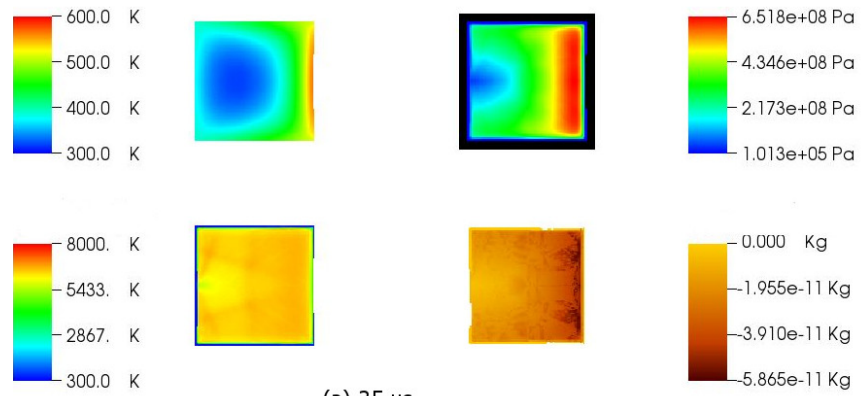

(a) $25 \mu \mathrm{s}$
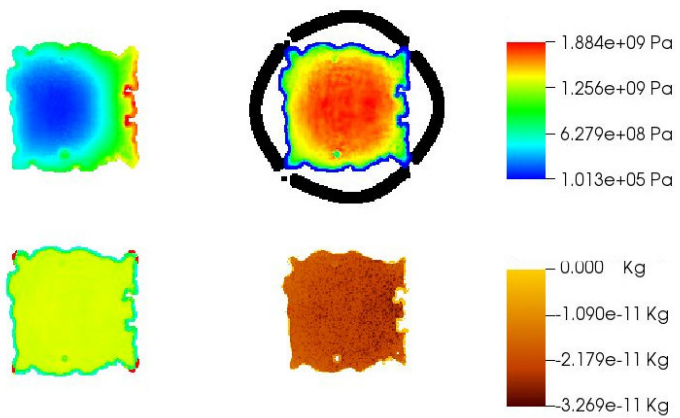

(c) $170 \mu \mathrm{s}$
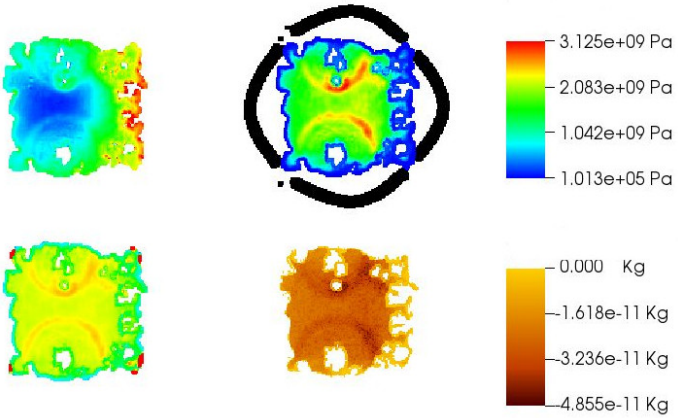

(e) $185 \mu \mathrm{s}$
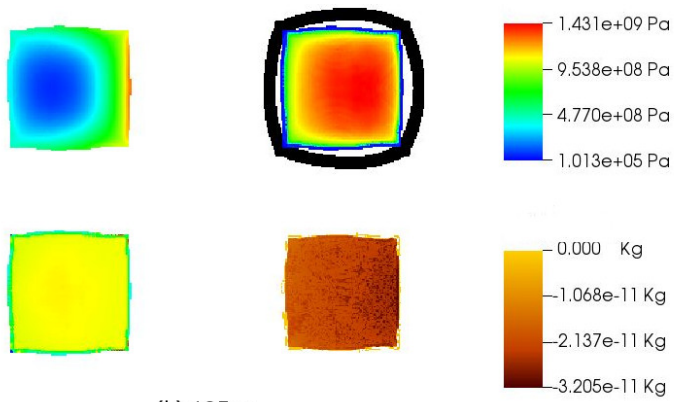

(b) $105 \mu \mathrm{s}$
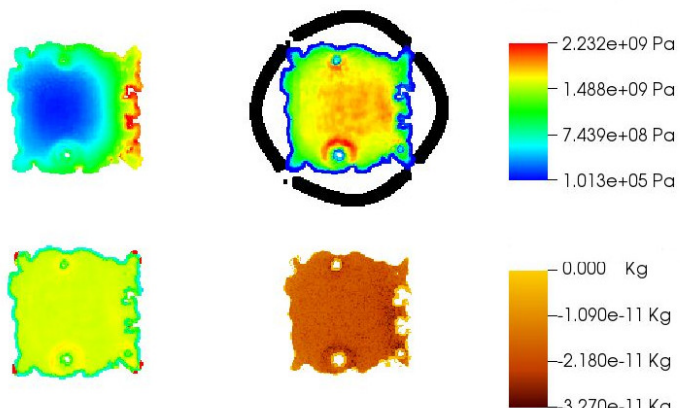

(d) $175 \mu \mathrm{s}$
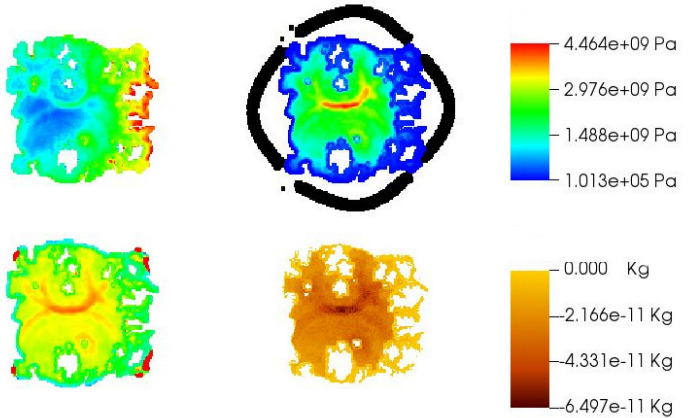

(f) $191 \mu \mathrm{s}$

Figure 6: A $85 \times 85 \times 1 \mathrm{~mm}$ device subject to $8.02 \mathrm{~kW} / \mathrm{m}^{2}$ heat flux on 1 side undergoing an ignition site detonation. Time reported is the amount of time after ignition.

detonation pressure, termed ignition site detonation (Figure $6)$. Ignition site detonation usually occurs at higher heating rates than seen with pressure induce detonation, where the temperature gradient of the energetic material is large. It takes about $25 \mu \mathrm{s}$ after ignition for convective burning to propagate through the entire device (Figure 6(a)) and about $105 \mu$ s for the maximum pressure to be confined to the center (Figure 6(b)). Since the condensed phase has a large temperature gradient, the product gas cools significantly within the solid during the duration of burning, reducing the amount of reactant going to product within the device. The velocity vector profile for this mechanism shows that the movement of the product gas is more inward than out. This can be due to the lower temperature of the PBX9501 in the center, reducing the reaction rate and causing a higher concentration of product gas to be produced from surface burning; therefore causing the gas to move inward.

About $170 \mu s$ after the beginning of deflagration, an ignition site forms, seen in Figure 6(c) on the bottom of the device seen in the reactant consumption plot. The formation mechanism of the ignition site is yet to be determined but the typical location is where the condensed phase temperature gradient is the steepest. At the ignition site, a "hole" is formed within the interior of the device and surface burning occurs releasing a large compaction wave. A standalone compaction wave has not yet been observed to initiate detonation. However, it is capable of producing multiple ignition sites within the wave itself. These new sites release waves congruently with the initial compaction wave and have been seen to transition into a detonation. The average amount of reactant going to product during a time-step in the reaction phase is $\sim 2 \times 10^{-11} \mathrm{~kg}$ per cell, and in the compaction waves it increased to $\sim 3.6 \times 10^{-11} \mathrm{~kg}$. In this simulation two pressure waves traveled towards the center (Figure 6(d-f)) until they coalesce and transition into detonation.

The crucial factor which seems to dictate the difference between the pressure detonation and the ignition cite detonation is the initial temperature gradient of the energetic material at the time of ignition and how it effects the temperature of the product gas during the reaction phase. In the 


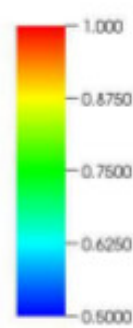

(a)

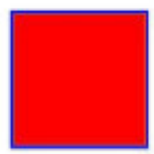

(b)

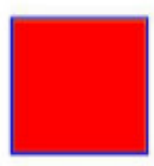

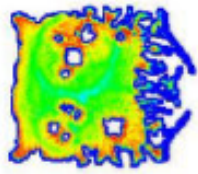

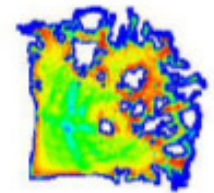

Figure 7: A volume fraction depiction of a $95 \times 95 \times 1 \mathrm{~mm}$ device prior to deflagration (left image) and the right image is after $220 \mu s$ of burning subject to a $50 \mathrm{~kW} / \mathrm{m}^{2}$ flux on one side (a) resulting in a detonation and two sides (b) causing a pressure burst.

middle range of high and low heat flux, a hybrid of the two mechanisms can be observed. In this scenario, the pressure profile and waves formed during the reaction phase are very similar to the pressure detonation mechanism. However, ignition sites begin to establish around the perimeter causing detonation to occur due to the coalescing waves. These different mechanisms attribute to the dependence of size and flux with respect to violence because of the temperature gradient that forms during the reaction phase; the higher the heat flux, the steeper the gradient. At higher heat fluxes and steeper gradients, the mechanism for detonation was due to the formation of ignition sites. However with smaller devices, ignition sites still occurred but the compaction waves were not as strong. Since smaller devices lack the length needed for a compaction wave to build the pressure needed to produce a stronger wave, a transition to detonation or a violent response will not occur. Compaction waves may still coalesce, but they will continue to travel throughout the charge damaging the remaining PBX9501. Once confinement has been breached the wave continues to expand away from the charge releasing the buildup of any pressure. Larger devices have the capacity for ignition sites to release compaction waves that may build up enough pressure after they coalesce and transition into a detonation. Therefore, the critical flux increases with device size because of the capability for a larger device to facilitate a detonation or an adequate length of unburnt material in which pressure can build up and transition.

The effect of the number of sides heated shows that the critical heat flux for two sided heating is less than one sided heating with the same device size. This observation can be in conjunction with the same effect that device size has on the critical heating flux. By heating two sides of the device, the burning of the energetic surfaces decreases the overall size of the device significantly more than subjecting the device to one side heating. This causes the compaction waves to not coalesce effectively and build to detonation pressures. Figure 7 shows the deterioration of two charges being subject to the same heat flux at the same time after ignition. The top device was subjected to heating on one side while the bottom device was heated on two sides. It is apparent that two sided heating causes greater damage to the device and thus mitigating the cook-off event to a case burst.

\section{CONCLUSIONS}

Two mechanisms responsible for deflagration to detonation transitions were observed in slow cook-off simulations of multiple device sizes; pressure detonation and ignition site detonation. In many respects these two mechanisms to detonation are the same, only differing in the origin of the pressure waves. With slow cook-off simulations the pressure waves originated from the center of the device forming a grid pattern transitioning to detonation when two of the wave collided producing pressures above $5 G P a$. While with faster cook-off simulations pressure waves originated from a cite with a high temperature gradient. Detonation then occurred from the colliding of two pressure waves. In both mechanisms, detonation occurred when two pressure waves collided and $\sim 17 \%$ PBX9501 was burned independent of device size, heat flux, and geometry.

From these studies it has been observed that when a device is exposed to a range of heat fluxes, the greater the flux, the more likely a mild explosion is going to occur. However as the device size increases, the likelihood of a transition into detonation also increases. This can be attributed to the ability of the device to sustain a pressure wave that will reach detonation pressures to transition into a violent event. This is dependent on the amount of unburnt material left in the device; the larger the device, the larger the area of unburnt material increasing the detonation. To reduce the risk of a transition to detonation, the device should to be heated on two sides instead of just one. This appears to reduce violence of reaction due to the lower critical heat flux and the increased surface burning on two sides. This in turn reduces the violence of reaction for the same device size.

\section{ACKNOWLEDGMENTS}

Joseph Peterson and John Schmidt are especially thanked. This work was supported by the National Science Foundation under subcontract No. OCI0721659. Uintah was developed by the University of Utah's Center for the Simulation of Accidental Fires and Explosions (C-SAFE) and funded by the Department of Energy, subcontract No. B524196. We would also like to thank TACC and NICS for access to computing resources.

\section{REFERENCES}

[1] K. Alexander, K. Gibson, and B. Baudler. Development of the Variable Confinement Cookoff Test. Technical report, Indian Head Division, Naval Surface Naval Surface Warfare Center, 1996.

[2] B. W. Asay and et al. Effect of Temperature Profile on Reaction Violence in Heated and Self-Ignited PBX-9501. In AIP Conference Proceedings, volume 620, pages 1065-1068, 2002.

[3] A. I. Atwood, P. O. Curran, D. Bui, and T. L. Boggs. Energetic Material Response in a Cookoff Model Validation Experiment. In 12th International Detonation Symposium, pages 975-984, 2003.

[4] M. Baer and J. Nunziato. A Two-Phase Mixture Theory for the Deflagration-to-Detonation Transition 
in Reactive Granular Materials. International Journal of Multiphase Flow, 12:861-889, 1986.

[5] S. Bardenhagen, J. Guilkey, K. Roessig, J. Brackbill, W. Witzel, and J. Foster. An Improved Contact Algorithm for the Material Point Method and Application to Stress Propagation in Granular Material. Computer Modeling in Engineering and Sciences, 2:509-522, 2001.

[6] W. Breshears. One-Dimensional Time-to Explosion (ODTX) in HMX Sphere. Technical Report LA-UR-98-1237, Los Alamos National Lab., NM, June 1997.

[7] P. Cheese, R. Briggs, J. Fellows, P. Haskins, and M. Cook. Cook-off Tests on Secondary Explosives. In 11th International Detonation Symposium, pages 272-278, Snowmass, CO, 1998.

[8] P. Dickson, B. Asay, B. Henson, and L. Smilowitz. Thermal Cook-Off Response of Confined PBX 9501. In The Royal Society A, volume 460, December 2004.

[9] P. Dickson, B. W. Asay, B. Henson, and C. Fugard. Observation of the Behavior of Confined PBX9501 Following a Simulated Cookoff Ignition. In 11th Symposium on Detonation, Snowmass, CO, 1998.

[10] J. Germain, A. Morris, S. Parker, A. Malony, and S. Shende. Performance Analysis Integration in the Uintah Software Development Cycle. International Journal of Parallel Programming, 31:35-53, 2003.

[11] J. Guilkey, T. Harman, and B. Banerjee. An Eulerian-Lagrangian Approach for Simulating Explosions of Energetic Devices. Computers and Structures, 85:660-674, 2007.

[12] B. Kashiwa. A Multifield Model and Method for Fluid-Structure Interaction Dynamics. Technical Report LA-UR-01-1136, Los Alamos National Laboratory, 2001.

[13] B. Kashiwa and E. Gaffney. Design Basis for cfdlib. Technical Report LA-UR-03-1295, Los Alamos National Laboratory, Los Alamos, 2003.

[14] B. Kashiwa and R. Rauenzahn. A Cell-Centered ICE Method for Multiphase Flow Simulations. Technical Report LA-UR-93-3922, Los Alamos National Laboratory, Los Alamos, 1994.

[15] M. Liberman, M. Kuznetsov, A. Ivanov, and I. Matsukov. Formation of the Preheated Zone Ahead of a Propagating Flame and the Mechanism Underlying the Deflagration-to-Detonation Transition. Physical Letters A, 373:501-510, 2009.

[16] J. Luitjens and M. Berzins. Improving the Performance of Uintah: A Large-scale Adaptive Meshing Computational Frameworking Computational Framework. In Proceedings of the 24th IEEE International Parallel and Distributed Processing Symposium (IPDPS10), 2010.

[17] J. Luitjens, M. Berzins, and T. Henderson. Parallel Space-Filling Curve Generation. Concurrency and Computation: Practice and Experience, 19:1387-1402, 2007.

[18] J. Maienschein, J. Wardell, R. Weese, and B. Cunningham. Understanding and Predicting the Thermal Explosion Violence of HMX-based and RDX-based Explosives-Experimental Measurements of Material Properties and Reaction Violence. In 12th
International Detonation Symposium, San Diego, California, 2002. Office of Naval Research.

[19] Q. Meng and M. Berzins. Scalable Large-scale Fluid-structure Interaction Solvers in the Uintah Framework via Hybrid Task-based Parallelism Algorithms. Sci tech report uusci-2012-004, University of Utah, 2012.

[20] Q. Meng, M. Berzins, and J. Schmidt. Using Hybrid Parallelism to Improve Memory Use in the Uintah Framework. In Proceedings of Teragrid 2011, page (published online), July 2011.

[21] Q. Meng, A. Humphrey, and M. Berzins. The Uintah Framework: A Unified Heterogeneous Task Scheduling and Runtime System. In Digital Proceedings of The International Conference for High Performance Computing, Networking, Storage and Analysis (SC12) - WOLFHPC Workshop. ACM, 2012.

[22] Q. Meng, J. Luitjens, and M. Berzins. Dynamic Task Scheduling for the Uintah Framework. In Proceedings of the 3rd IEEE Workshop on Many-Task Computing on Grids and Supercomputers (MTAGS10), 2010.

[23] S. Parker, J. Guilkey, and T. Harman. A Component-based Parallel Infrastructure for the Simulation of Fluid Structure Interaction. Engineering with Computers, 22(3-4):277-292, 2006.

[24] W. Perry, P. Dickson, G. Parker, and B. W. Asay. Quantification of Reaction Violece and Combustion Enthalpy of Plastic Bonded Explosive 9501 Under Strong Confinement. Journal of Applied Physics, 97:1-8, 2005.

[25] J. Peterson and C. Wight. An Eulerian-Lagrangian Computational Model for Deflagration and Detonation of High Explosives. Combustion and Flame, 159:2491-2499, 2012.

[26] H. Sandusky, G. Chambers, W. Erikson, and R. Schmitt. Validation Experiments for Modeling Slow Cook-Off. In 12th International Detonation Symposium, pages 863-872, Office of Naval Research, Arlington, VA, 2003.

[27] J. Schmidt, M. Berzins, J. Thornock, T. Saad, and J. Sutherland. Large Scale Parallel Solution of Incompressible Flow Problems using Uintah and hypre. In Proceedings of CCGrid 2013. IEEE/ACM, 2013.

[28] D. Sulsky, S. Zhou, and H. Schreyer. Application of a Particle-in-cell Method to Solid Mechanics. Computer Physics Communications, 87:236-252, 1995.

[29] C. M. Tarver and S. K. Chidester. On the Violence of High Explosive Reactions. Journal of Pressure Vessel Technology, 127:39-48, February 2005.

[30] L. Tran and M. Berzins. IMPICE Method for Compressible Flow Problems in Uintah. International Journal For Numerical Methods In Fluids, 2011.

[31] J. Wardell and J. Maienschein. The Scaled Thermal Explosion Experiment. In 12th Internationsl Conference Symposium, San Diego, California, July 2002.

[32] C. Wight and E. Eddings. Science-Based Simulation Tools for Hazard Assessment and Mitigation. Advancements in Energetic Materials and Chemical Propulsion, 114:921-937, 2008. 